\title{
A LOWER LIMIT TO THE EXCESS OF COMPANIONS AMONG
} SEYFERT GALAXIES

\author{
P. RAFANELLI and M. VIOLATO \\ Department of Astronomy, University of Padova, Italy
}

It is not yet clear which is the role played by interaction on Seyfert activity. Presently there are on this topic contradictory statistical results, based on the study of more or less rich samples of Seyfert galaxies. Petrosian (1982), Dahari (1985) and Mac Kenty $(1989,1990)$ found an excess of Seyferts among galaxies which have nearby companions and an excess of galaxies with nearby neighbors among Seyfert galaxies. The contrary result was found by Bushouse (1987) and by Fuentes Williams \& Stocke (1988). In order to find more reliable conclusions, taking advantage of the increased number of Seyfert galaxies identified in the last years, we have applied the statistical procedures used by Dahari (1985) to a sample of 287 Seyfert-1 (S1) and 195 Seyfert-2 (S2) (namely to all known S1 and S2 galaxies with $z \leq 0.11$ and $\delta \geq-23^{\circ}$ ). A subsample has been extracted carefully excluding objects, identified as Seyfert on the basis of their morphology ( e.g. on the basis of the presence of a companion) and brighter than $m_{B}=15.5$, the magnitude at which the cumulative number of S1 and S2 galaxies becomes flat. The possible companions of our Seyfert galaxies have been identified on the blue POSS prints by visual inspection looking for objects not more distant than 3 diameters from the Seyfert galaxy and not more than 3 magnitudes fainter. It results that $\sim 35 \%$ of both the S1 and S2 galaxies have a neighbor. This percentage is reduced to $\sim 14 \%$ for both classes of galaxies excluding the possible optical companions, the upper limit to the number of which has been derived starting from the counts of galaxies published by Shane and Wirtanen (1967), assuming that the probability of finding an optical companion is a Poisson probability. The same procedure applied to a control sample of 281 normal galaxies indicates that only $\sim 3 \%$ of the galaxies have a close companion. In conclusion Seyfert galaxies show a larger percentage (14\%) of objects with companion than normal galaxies do (3\%). This excess, which is the same for S1 and S2 galaxies, has been derived overestimating the number of optical companions and its value is then a lower limit.

\section{References}

Bushouse, H.A.: 1987, ApJ, 320, 49

Dahari, O.: 1985, ApJS, 57, 643

Fuentes Williams, T., Stocke, J.T.: 1988, $A J$, 96, 1235

MacKenty, J.W.: 1989, $A p J, 343,125$

MacKenty, J.W.: $1990, A p J S, 72,231$

Petrosian, A.R.: 1982, Astrofizika, 18, 548 (English trans. Astrophysics, 18, 312)

Shane, C.D., Wirtanen, C.A.: 1967, Publ. Lick Obs., Vol. 22, Part I, University of Chicago, Chicago Ilinois, 647

T. J.-L. Courvoisier and A. Blecha: Multi-Wavelength Continuum Emission of AGN, 519.

C 1994 IAU. Printed in the Netherlands. 